\title{
Heterogeneous Configuration: A Better Approach to Analyze VANET
}

\author{
Pankaj Joshi \\ M.Tech Scholar, ECE \\ SBSSTC, Moga Road \\ Ferozepur - 152004
}

\author{
Jaswinder Kaur \\ Assistant Professor, ECE \\ SBSSTC, Moga Road \\ Ferozepur - 152004
}

\author{
Inderjeet Singh Gill \\ Assistant Professor, ECE \\ SBSSTC, Moga Road \\ Ferozepur - 152004
}

\begin{abstract}
A Vehicular Ad-hoc Network (VANET) is an area of wireless technologies that has the property of high node mobility and dynamic topology. In this paper we focus on technology which has major role in avoiding accidents using homogeneous and heterogeneous configurations in different VANET scenarios. For this purpose OMNET++ and VEINS (Vehicles in Network Simulation) is used as the developing platform. Simulation shows that heterogeneous configuration achieve better performance than homogeneous configuration when compared in four different node densities i.e. $20,30,40$ and 50 nodes in terms of back off time, sent packet, lost packet and busy time.
\end{abstract}

\section{Keywords}

Vehicular Ad-hoc Networks (VANET), Road Side Unit (RSU), Vehicle to Vehicle (V2V) and Vehicle to Infrastructure (V2I)

\section{INTRODUCTION}

Wireless communication has influenced ours lives in many ways by making our life more convenient, easier and comfortable. VANET is the emerging technology which is growing rapidly in the field of communication technologies. It is the sub form of mobile Ad-hoc networks (MANET) in which nodes is defined as vehicles and have organized mobility pattern. Vehicular Ad-Hoc Network can be defined as a highly mobile Ad-Hoc Network between vehicles, road side units, and other infrastructure network. VANET mainly consists of vehicles and RSUs which are the essential entities supporting it. Each vehicle is equipped with an on board units (OBU) used for exchanging the information with other OBUs and RSUs. Vehicles are also fitted with hardware such as sensors, GPS device and omni directional antenna that allow detailed information about vehicle to transmit to other vehicles and RSUs. The application unit is a device that uses applications provided by the network and has liability of mobility and networking functions. RSU: RSUs are fixed infrastructural unit along the roadside or at specified locations. RSU is equipped with network devices which are used for dedicated short range communication and communication within the infrastructural network. RSUs collect the information from one network and forward the information to another network $[1,2]$.

\subsection{VANET Communication}

\subsubsection{Inter-Vehicle Communication}

Inter-Vehicle Communication: IVC system is categorized in two configuration i.e. Single-hop and multi-hop IVCs (SIVCs and MIVCs). When a vehicle directly communicate with other vehicle using a wireless connection, they form a single hop inter vehicle communication. SIVC systems are useful for applications requiring short-range communication. In MIVCs, there is no direct connection between the source and destination vehicle, the information will be multi hop transmitted over the vehicles and then reach the destination vehicle. MIVC systems are more complex than SIVCs but can also support applications that require long-range communication.

\subsubsection{Roadside-Vehicle Communication}

Roadside - Vehicle Communication: The vehicle-to-roadside communication configuration represents a single hop broadcast where the roadside unit sends broadcast information to all vehicles in its surrounding area. The vehicle communicates with RSU for sending, forwarding and receiving information from one node to another. RSU is further connected to infrastructural network and therefore allows the vehicles to access the internet for non safety applications. Vehicle-to-roadside communication provides a high bandwidth link and enables high data rate transmission between vehicles and roadside units [3-6].

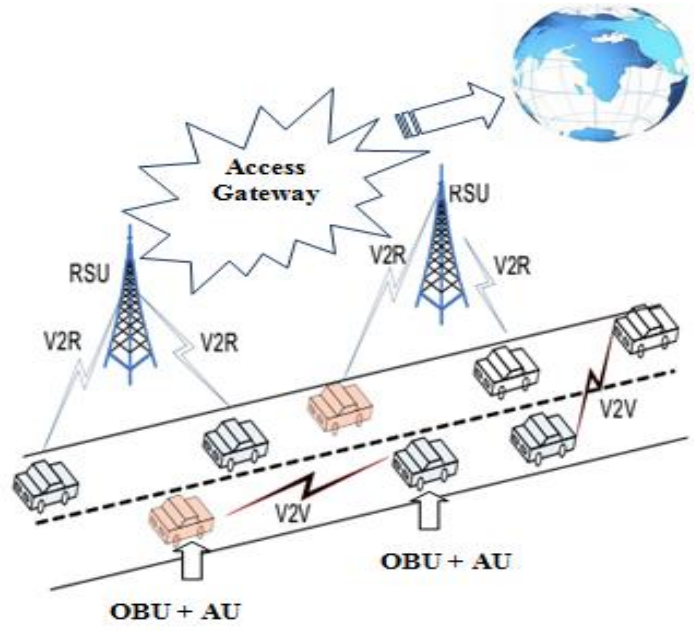

Fig 1: Architecture of VANET

\subsection{VANET Applications}

Vehicle to vehicle and vehicle to infrastructure communications have stimulated a wide variety of applications that led to improvement of road safety, comfort to drivers and passengers and traffic optimization. VANET applications are categorised into:

\subsubsection{Safety Applications}

These applications intend to save people's lives and provide safe environment. The Vehicle Safety Communication (VSC) project indicates possible safety applications for DSRC and is 
categorised according to their effectiveness in avoiding accidents. Some safety messages are transmitted periodically while other messages are transmitted when a specified event occurs. The safety applications includes Traffic Signal Violation Warning, Curve Speed Warning, approaching emergency vehicle warning, Emergency Electronic Brake Lights, Pre crash Warning, Wrong Way driving warning, Cooperative Forward Collision Warning, Left Turn Assistance, Stop Sign Movement Assistance, Lane Change Warning, road condition warning, Cooperative Collision Warning and Intersection Collision Warning [7].

\subsubsection{Non Safety Applications}

This application aims to enhance traffic efficiency and increase the comforts of drivers and travellers. The non safety applications includes Access Control, Gas Payment, Point-ofInterest Notification, weather information, specific locations like restaurants, petrol pumps, hotels etc, play online games, Data Transfer, Instant Messaging, Car Rental, Enhance Route Guidance, Data Transfer, Parking Lot Payment, Toll Collection and access internet [8].

\section{VANET RELATED PROJECTS}

All Many researchers are involved in national and international consortia related to different challenges in VANET which results in large number of projects. Several automobile companies like BMW, Ford, Nissan, Toy ota etc participate in Vehicle Safety Communications Consortium [9]. Brief views of selected projects linked with VANET are

1. FleetNet - A pioneer research project to develop a platform for inter-vehicle communication which provides information services and safety applications [10].

2. PReVENT - A R\&D project that follow general concepts: virtual safety belt, time to collision and layered architecture. PReVENT project is the major initiative on road safety by the European Commission [11].

3. SeVeCom [12] - An EU project focusing on the security and privacy issues in vehicular communication. A large number of security threats are identified and removed from sy stem to provide secure communication.

4. Co-operative Vehicle-Infrastructure Sy stems (CVIS) - A project which is focused on vehicle to vehicle and V2I communication and supports cooperative applications [13].

5. SAFESPOT - A project designing Safety Margin Assistant which should help to detect in advance the accident before it may occur. The new safety applications are developed based on cooperative approach.

6. Network on Wheels (NOW) - NOW is German research project which is successor of FleetNet. The main objectives are to solve technical issues on communication protocols and data security for vehicle to vehicle communication sy stem [14].

7. Car talk 2000 - It was three year project which have main focus on Information and warning functions like traffic load, collision avoidance and road accident notifications [15].

8. Advanced Safety Vehicle Program (ASV-2), (ASV-3) (ASV-4) - A project supported by Japanese Ministry of Transport which implements the active and passive safety procedures in vehicular environment.

\section{DEDICATED SHORT RANGE COMMUNICATION (DSRC)}

Please In 1991, the US Congress passed the Intermodal Surface Transportation Efficiency Act of 1991 that incorporate technology into the transportation infrastructure to improve safety. The first generation of the Intelligent Transportation System (ITS) is Dedicated Short-Range Communication (DSRC) system operates at $915 \mathrm{MHz}$ and has a transmission rate of $0.5 \mathrm{Mb} / \mathrm{s}$. The example of a first generation DSRC application is EZ Pass that is used for electronic toll collection which had limited success. In October 1999, the FCC allocated the $75 \mathrm{MHz}$ of bandwidth in the $5.9 \mathrm{GHz}$ band for the second generation of DSRC which has been used for inter-vehicle and vehicle-to-infrastructure (V2I) communications. DSRC provides high data rates within small communication range. At the PHY and MAC layers DSRC utilizes Wireless Access for Vehicular Environments (WAVE), which is a conjunction of the standards IEEE 802.11p and IEEE 1609.x to provide wireless access in vehicular environments. The WAVE provides a multichannel control mechanism to operate seven $10-\mathrm{MHz}$ wide channels defined in the DSRC spectrum. One is the control channel $(\mathrm{CCH})$ for common safety communications only, and the remaining six are known as the service channels ( $\mathrm{SCHs}$ ) for other non safety applications. DSRC/WA VE supports a situation in which the vehicles can move at a speed of 200 kmph with transmission rate of $6 \mathrm{Mb} / \mathrm{s}$ to $27 \mathrm{Mb} / \mathrm{s}$. DSRC offers high throughput and have transmission range up to $1000 \mathrm{~m}$. The data rate can reach up to $54 \mathrm{Mb} / \mathrm{s}$ if two service channels are combined to form one $20 \mathrm{MHz}$ channel. In the middle of the stack, DSRC employs a suite of standards defined by the IEEE 1609 Working Group: 1609.4 for Channel Switching, 1609.3 for Network Services and 1609.2 for Security Services [17-19].

\section{EXPERIMENTAL SETUP}

The simulation model consists of different nodes and 25 roadside units based on the dedicated short range communication. In this model, the homogeneous and heterogeneous configurations are analysed in grid view of road having parallel lanes. A homogeneous configuration is defined as the scenario in which the nodes are moving with constant speed i.e. $28 \mathrm{mps}$ and for heterogeneous configuration the vehicles are moving at different speeds $(25-35 \mathrm{mps})$ throughout the simulation. We evaluate the performance parameters of different network configuration by changing the number of vehicles and randomly positioning the vehicles over the entire simulation area. OMNeT++ is an object-oriented modular discrete event system simulation framework used for modelling communication networks and protocols, queuing networks, and other distributed hardware systems. The Veins framework includes suite of models which make simulations of vehicular network as practical as possible [20]. The Connection Manager module controls all connections between the nodes and periodically communicates with the mobility module and Channel Access. For whole network the basic utility module used is Base World Utility that provides utility methods and information for the network as well as simulation functionality. The vehicle is a host module using $802.11 \mathrm{p}$ Network interface card. The homogeneous and heterogeneous configurations are compared in four different scenarios where the vehicle originates randomly and node density varies as 20, 30, 40 and 50 respectively. The fig. 2 shows the simulation model of homogeneous and heterogeneous configurations and fig. 3 presents the simulation model with 50 nodes. 


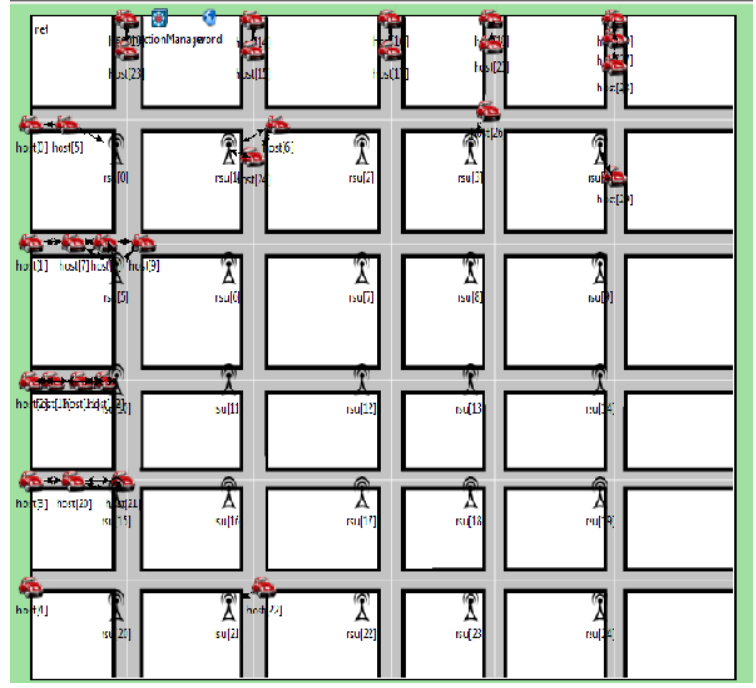

Fig. 2 Simulation model of homogeneous and heterogeneous configurations with 30 nodes

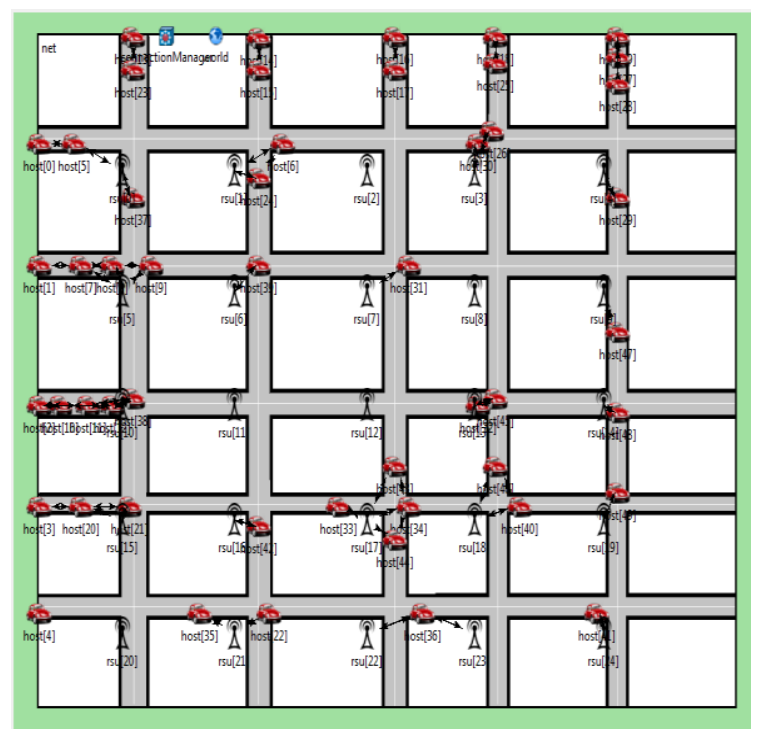

Fig. 3 Simulation model of homogeneous and heterogeneous configuration with 50 nodes

The parameters used in homogeneous and heterogeneous configuration for simulating the model are given in the table1.

\section{RESULTS AND DISCUSSIONS}

\subsection{Sent Packets}

Sent packets are the total number of packets sent during the communication between the vehicles and vehicle to roadside units. In case of homogenous configuration the sent packets are greater than the heterogeneous configuration because in former case the some vehicles are equidistant from each other and remain in communication for whole simulation whereas in latter case, the vehicles are moving with different speeds and one vehicle may cross other vehicles and not involved in communication with same vehicle for entire simulation. So in heterogeneous configuration, more packets can be sent for other applications.
Table 1 Simulation Parameters of Network

\begin{tabular}{|c|c|c|}
\hline $\begin{array}{c}\text { Sr. } \\
\text { no. }\end{array}$ & \multicolumn{2}{|c|}{ Simulation Parameters } \\
\hline 1 & Simulator & $\begin{array}{c}\text { OMNET++, } \\
\text { Veins } 2.1\end{array}$ \\
\hline 2 & Simulation Area & $10 x 10 \mathrm{Km}$ \\
\hline 3 & Simulation Time & $200 \mathrm{sec}$ \\
\hline 4 & Maximum Transmission Power & $20 \mathrm{~mW}$ \\
\hline 5 & Mac Bit Rate & $18 \mathrm{Mbps}$ \\
\hline 6 & Thermal Noise & $-110 \mathrm{dBm}$ \\
\hline 7 & Physical Layer Sensitivity & $-94 \mathrm{dBm}$ \\
\hline 8 & Carrier Frequency & $5.8 \mathrm{GHz}$ \\
\hline 9 & Variable node densities & $20,30,40,50$ \\
\hline 10 & Number of RSUs & 25 \\
\hline 11 & Vehicle Speed(Homogeneous) & $28 \mathrm{mps}$ \\
\hline 12 & Vehicle Speed (Heterogeneous) & $25-35 \mathrm{mps}$ \\
\hline
\end{tabular}

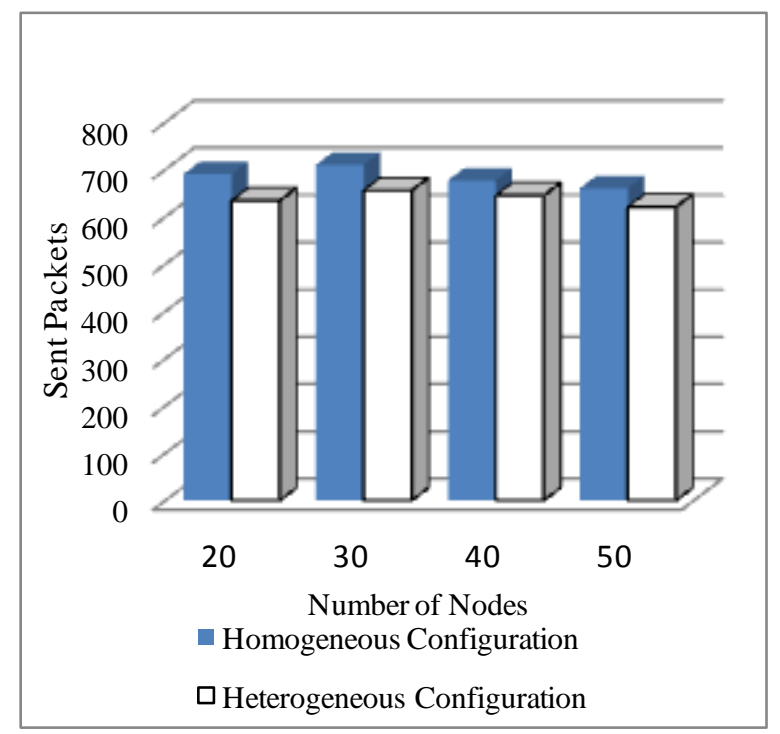

Fig. 4 Sent Packets

\subsection{Times into Back off State}

When a vehicle experienced a collision on a network, it waits for some specified time and then again try to retransmit. When the vehicle finds the channel busy, it chooses a random back off time and transmits only when back off time is elapsed. As the vehicle involves much in communication, the time in back off state is also more. In homogeneous configuration, the vehicles involves in communication for much larger time so the times the vehicles go to back off state is also more as compared to heterogeneous configuration. In case of heavily loaded network, the back off period is increased to prevent the repeated collisions. 


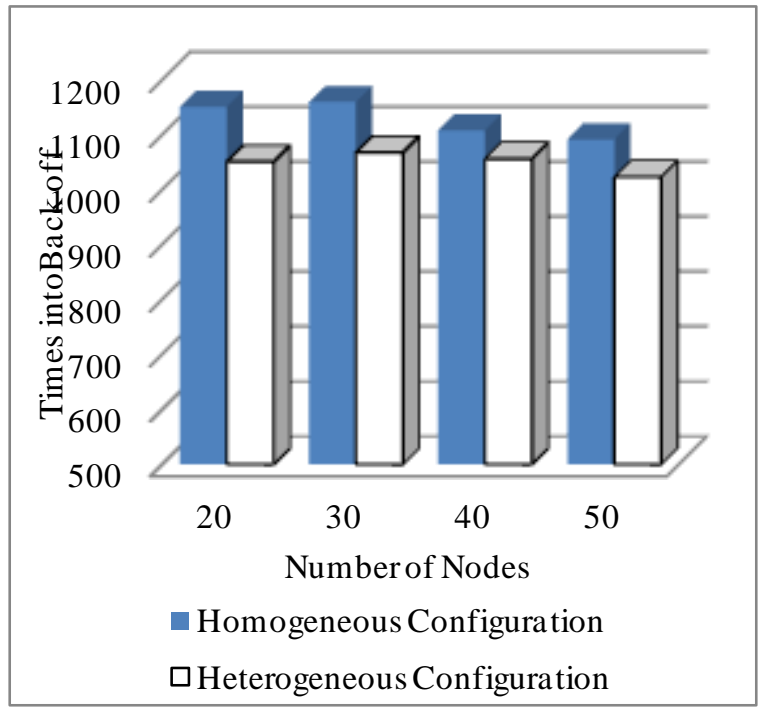

Fig. 5 Times in Back off S tate

\subsection{Total Lost Packets}

The Total Lost Packets defined as the total number of packets dropped during the simulation. The graph shows the number of packets dropped when one vehicle undergo vehicle to vehicle communication and vehicle to infrastructure communication. As vehicle communicates with more than two nodes and road side unit, the probability of packet lost increases. The heterogeneous configuration performs much better than homogeneous network in regards with the lost packets parameter. In heterogeneous networks, the chance of busy channel is less as the probability of a vehicle to communicate with other vehicles and RSU for a particular time is less.

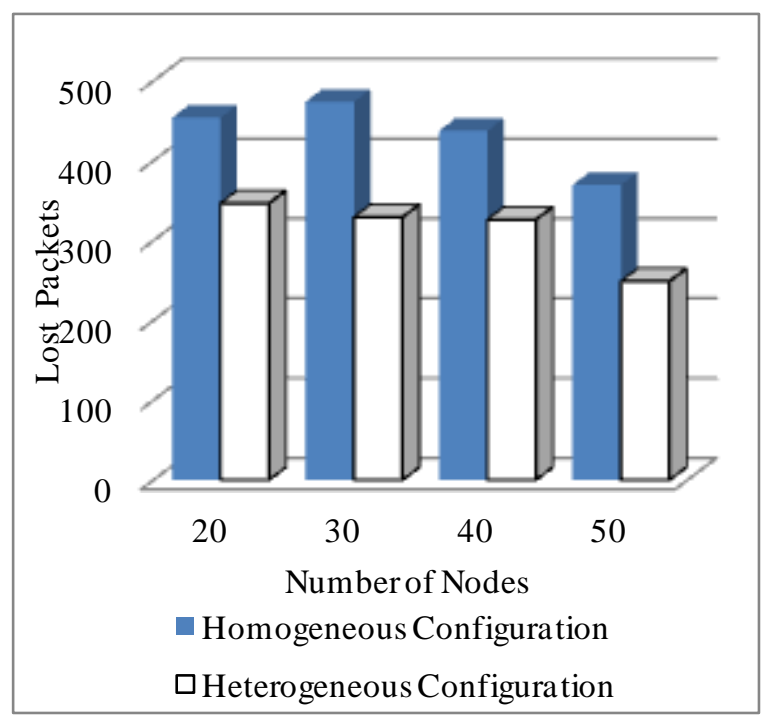

Fig. 6 Lost Packets

\subsection{Busy Time}

Busy time is defined as the time involved in vehicle to vehicle communication and vehicle to infrastructure by a particular vehicle. If the value of busy time is more means that a vehicle is engaged more in communication either with other vehicles or with road-side units. So busy time depends upon the number of vehicles communicate on the way to their destination. So as the communication between the vehicles or the road side units continues, the busy time of that vehicle is considered to be more than the other nodes. In homogeneous networks the vehicles are moving with constant speed and will remain in communication once connected so they have more busy time as compared to heterogeneous networks. The heterogen eous networks have extra time which can be utilize for sending other safety related messages between the vehicles and RSUs.

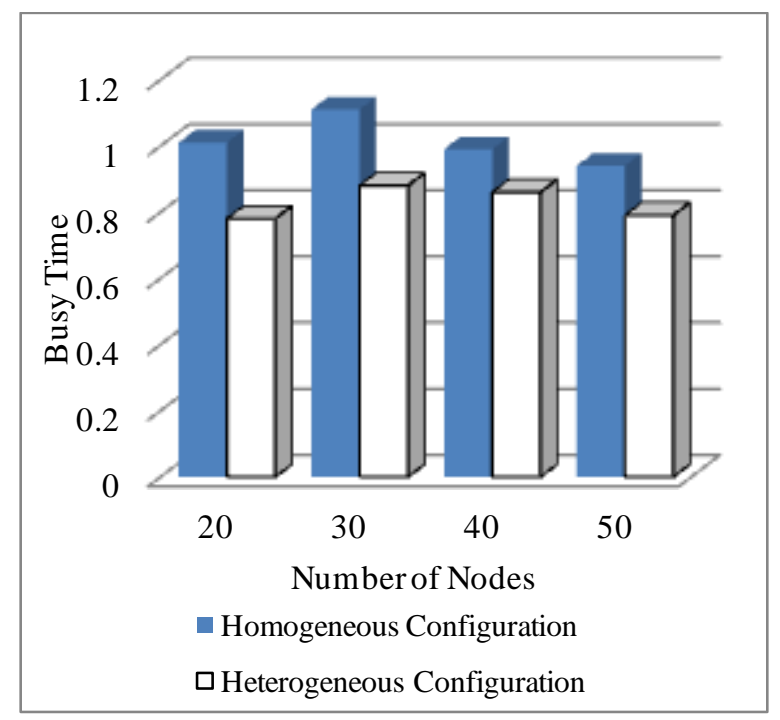

Fig. 7 Busy Time

\section{CONCLUSION AND FUTURE SCOPE}

In our Research work, the vehicle to vehicle and vehicle to infrastructure communication is implemented using dedicated short range communication. The two different configurations are analy sed and compared in four different node densities i.e. $20,30,40$, and 50 nodes with respect to different performance parameters. The simulation results show that the heterogeneous configuration has better performance than homogeneous configuration for analysing VANET scenario. The packet loss in heterogeneous configuration is lesser than homogeneous configuration and other parameters back off time and busy time are also small. Due to good system parameter values, the other safety applications will be easily added in heterogeneous configuration. Hence the heterogeneous configuration will enhance results in VANET applications.

\section{REFERENCES}

[1] Olariu and Weigle, 2009 C.C. Communication Consortium; Ieee Trial-use Standard for Wireless Access in Vehicular Environments

[2] Sok-Ian Sou; Tonguz, O.K. , Oct 2011 "Enhancing VANET Connectivity Through Roadside Units on Highways" Vehicular Technology, IEEE Transactions, Vol-60, Issue: 8, ISSN :0018-9545

[3] Saif Al-Sultann, Moath M. Al-Doori, Ali H. Al-Bay atti, Hussien Zedan, February 2013 "A comprehensive survey on vehicular Ad Hoc network", Journal of Network and Computer Applications Elsevier.

[4] Zaydoun Yahya Rawashdeh and Syed Masud Mahmud 2011, Communications in Vehicular Ad HocNetworks, Mobile Ad-Hoc Networks: Applications, Prof. Xin Wang (Ed.), ISBN: 978-953-307-416-0, InTech,Available from: http://www.intechopen.com/books/mobile-ad-hocnetworks-applications/communications-invehicular-adhoc-networks 
[5] Sherali Zeadally·Ray Hunt Y Yuh-Shyan Chen-Angela Irwin-Aamir Hassan 2010, Vehicular ad hoc networks (VANETS): status, results, and challen ges Springer

[6] Schoch, E. Kargl, F. Weber, M. Leinmuller, T, Nov 2008 "Communication patterns in VANETs", Communications Magazine, IEEE, Vol-46, Issue: 11, 119-125.

[7] Khalid Abdel Hafeez, Lian Zhao, Bobby Ma, and Jon W. Mark September 2013 "Performance Analysis and Enhancement of the DSRC for VANET's Safety Applications", IEEE Transactions on Vehicular Technology, Vol. 62, NO. 7.

[8] Moustafa, H. \& Zhang, Y. 2009, Vehicular networks: Techniques, standards, and applications, Auerbach Publications Boston, MA, USA.

[9] Vehicle Safety Communications Consortium 2005, consisting of, BMW, DaimlerChrysler, Ford, GM, Nissan, Toy ota, and VW. Vehicle safety communications project task 3 final report: Identify intelligent vehicle safety applications enabled by DSRC.

[10] Ranz, W., Eberhardt, R. \& Luckenbach, T. 2001, Fleetnet-internet on the road, Proc. 8th World Congress on Intelligent Transport Systems.

[11] Schulze, M., Nocker, G. \& Bohm, K. 2005, PReVENT: A European program to improve active safety, Proc. of 5th International Conference on Intelligent Transportation Systems Telecommunications, France.

[12] Leinmüller, T., Butty an, L., Hubaux, J., Kargl, F., Kroh, R., Papadimitratos, P., Raya, M. \& Schoch, E. 2006, $\mathrm{SeVeCOM}$ - secure vehicle communication, Proceedings of IST Mobile Summit.

[13] CVIS (Cooperative Vehicles and Infrastructure Systems) 2010, www.cvisproject.org.
[14] Festag, A., Noecker, G., Strassberger, M., Lübke, A., Bochow, B., Torrent-Moreno, M., Schnaufer, S., Eigner, R., Catrinescu, C. \& Kunisch, J. 2008, Now-network on wheels: Project objectives, technology and achievements, Proceedings of 6th International Workshop on Intelligent Transportations (WIT), Hamburg and Germany .

[15] Reichardt, D., et al. 2002, CARTALK 2000 safe and comfortable driving based upon inter-vehiclecommunication. In Proceedings of IEEE intelligent vehicle symposium, Vol. 2, pp. 17-21.

[16] Yuan Yao; Lei Rao; Xue Liu, Oct 2013 "Performance and Reliability Analysis of IEEE 802.11p Safety Communication in a Highway Environment", IEEE Transactions on Vehicular Technology, Vol-62, Issue: 9 pp 4198 - 4212 ISSN: 0018-9545.

[17] John B. Kenney, July 2011 “ Dedicated Short-Range Communications (DSRC) Standards in the United States", Proceedings of the IEEE, Vol. 99, No. 7.

[18] FCC, Spectrum policy task force report 2002, ET Docket No. 02-135.

[19] Sebastian Grafling, Petri Mahonen, Janne Riihijarvi 2010 "Performance evaluation of IEEE 1609 WAVE and IEEE 802.11 p for vehicular communications", in: 2010 Second International Conference on Ubiquitous and Future Networks (ICUFN),IEEE, pp. 344-348.

[20] OMNET++ user manual and guide (http://www.omnetpp.org/documentation) and VEINS (veins.car2x.org)

[21] Hannes Hartenstein, Kenneth P Laberteaux, 2010 "VANET: Vehicular Applications and Inter-Networking Technologies" handbook intelligent transportation systems. 